\title{
IMOBILIZAÇÃO DE COMPLEXO DE RUTÊNIO CONTENDO BASE DE SCHIFF NA UNIDADE METALO- ORGÂNICA UiO-67
}

\author{
Herculys B. Jorge, Cleiser T. P. da Silva, Helena S. Nakatani, Andrelson W. Rinaldi e Vagner R. De Souza* \\ Departamento de Química, Universidade Estadual de Maringá, 87020-900 Maringá - PR, Brasil
}

Recebido em 28/01/2018; aceito em 17/04/2018; publicado na web em 22/05/2018

\begin{abstract}
IMMOBILIZATION OF RUTHENIUM SCHIFF BASE COMPLEX IN THE METAL-ORGANIC FRAMEWORK UIO-67. Zirconium-based metal-organic framework, UiO-67, was synthesized and used as support for the immobilization of the $\left[\mathrm{RuCl}_{2}(\mathrm{ENAPY})\right]($ ENAPY = N,N'-bis(7-metyl-2-pyridylmethylene)-1,2-diiminoethane)). The immobilized system exhibited violetblue color. The $\left\{\mathrm{UiO}-67 /\left[\mathrm{RuCl}_{2}(\mathrm{ENAPY})\right]\right\}$ was characterized by cyclic voltammetry, X-ray diffraction, UV-Vis, FTIR and X-ray photoelectron spectroscopy. The electrochemistry of the immobilized system is characterized by a reversible pair of waves at $+0.40 \mathrm{~V}$ (vs NHE), ascribed to the $\mathrm{Ru}^{3+2+}$ redox process. The electronic spectrum of the immobilized system is characterized by bands at 520 and $380 \mathrm{~nm}$ ascribed to a ligand-to-metal charge-transfer transitions $\left\{\operatorname{Ru}^{2+}\left(\mathrm{d}_{\pi}\right) \rightarrow \operatorname{ENAPY}\left(\mathrm{p}_{\pi}^{*}\right)\right\}$. The IR bands of immobilized complex $\left\{1630 \mathrm{~cm}^{-1}\left(\mathrm{v}_{\mathrm{asc}=\mathrm{C}}\right), 1590 \mathrm{~cm}^{-1}\left(\mathrm{v}_{\mathrm{asC}=\mathrm{N}}\right), 1450 \mathrm{~cm}^{-1}\left(\mathrm{v}_{\mathrm{sC}=\mathrm{C}}\right), 1340 \mathrm{~cm}^{-1}\left(\mathrm{v}_{\mathrm{sC}=\mathrm{C}}\right)\right\}$ are weak in comparison with the neat complex due to their low concentration in MOF cages. X-ray diffractogram of the immobilized system indicated that the crystallinity and morphology of UiO-67 was preserved during inclusion of ruthenium complex. The XPS spectra of UiO-67 and UiO-67/ruthenium complex shows a signal at $462 \mathrm{eV}$ that can be ascribed to $\mathrm{Ru} 3 \mathrm{p}^{3 / 2}$ and at $400 \mathrm{eV}$ ascribed to $\mathrm{N} 1 \mathrm{~s}$ core level of the ruthenium Schiff base complex immobilized into MOF.
\end{abstract}

Keywords: porous materials; post-synthetic modification; N4 donor ligand; spectroscopic properties.

\section{INTRODUÇÃO}

Com base em princípios elementares da química de coordenação e no aproveitamento adequado das características eletrônicas e estéricas dos íons metálicos e ligantes, foram projetadas estruturas moleculares relativamente complexas classificadas como polímeros de coordenação ou metal organic framewoks (MOF). ${ }^{1}$

$\mathrm{Na}$ construção de polímeros baseados em compostos de coordenação, o acoplamento interunidades pode ser efetuado por meio de ligantes ou complexos de ponte capazes de formar estruturas mono, bi ou tridimensionais. ${ }^{2}$ Sistemas lineares ou unidimensionais podem ser desenvolvidos com sais de cobalto(II) e ligantes 4,4'-bipiridina, em solução aquosa. ${ }^{3}$ Sistemas bidimensionais são obtidos pela polimetalação de estruturas planares com propriedades multiponte como as meso-tetrapiridilporfirinas de cobre. ${ }^{4}$ Finalmente, sistemas tridimensionais podem ser obtidos pela polimetalação de complexos baseados no tris(bipiridina)rutênio(II), $\left[\mathrm{Ru}(\mathrm{bpy})_{3}\right]^{2+}$, e sistemas poli-imínicos substituídos com grupos coordenantes. ${ }^{5}$ As vantagens de sistemas tridimensionais estão associadas ao desenvolvimento de materiais com elevada porosidade, com poros uniformes e ordenados em escala molecular, bem como na estabilidade dessas estruturas após a remoção da maior parte das moléculas do solvente presentes nos poros desse material. ${ }^{6}$

Considerando o potencial inesgotável dos compostos de coordenação na construção de estruturas metalo-orgânicas (MOFs), Cavka e colaboradores ${ }^{7}$ reportaram a síntese de uma nova classe de polímeros de coordenação tridimensional baseada em óxido de zircônio (IV) conectado por moléculas de ácidos dicarboxílicos. Como os primeiros derivados desta classe foram obtidos na Universidade de Oslo, as novas estruturas metalo-orgânicas foram designadas de $\mathrm{UiO}$, em homenagem à instituição de pesquisa.

Dentre as MOFs do tipo UiO, destacamos a UiO-67 que é constituída por clusters octaédricos de $\left[\mathrm{Zr}_{6} \mathrm{O}_{4}(\mathrm{OH})_{4}\right]$ ligados a doze

*e-mail: vrsouza2@uem.br moléculas de ácido 4,4'-bisfenildicarboxílico (BPDC), formando uma estrutura supramolecular organizada, simétrica e tridimensional, com cavidades octaédricas de diâmetro $20 \AA ̊$ e cavidades tetraédricas de diâmetro de $\sim 12$ Å. ${ }^{8}$ Essa rede cristalina é mantida mesmo quando a MOF é submetida à temperatura de $300^{\circ} \mathrm{C}$, como mostra o estudo de estabilidade térmica desenvolvido por Cavka e colaboradores. ${ }^{7}$ Segundo os autores, o aquecimento da amostra no intervalo de temperatura $250-300{ }^{\circ} \mathrm{C}$ faz com que o cluster $\mathrm{Zr}_{6} \mathrm{O}_{4}(\mathrm{OH})_{4}$ perca duas moléculas de água e seja convertido em $\mathrm{Zr}_{6} \mathrm{O}_{6}$. O cluster $\mathrm{Zr}_{6} \mathrm{O}_{6}$, formado pela desidratação do precursor, continua conectado às moléculas de ácido 4,4'-bisfenildicarboxílico preservando a porosidade da estrutura metalo-orgânica. A área superficial da UiO-67 pode variar de 500 a $2000 \mathrm{~m}^{2} \mathrm{~g}^{-1}$, dependendo do método de ativação e do solvente utilizado na preparação da MOF, visto que a metodologia aplicada pode provocar imperfeições estruturais associadas à ausência de molécula de ácido 4,4'-bisfenildicarboxílico na esfera de coordenação do centro metálico, ${ }^{9,10}$ conforme representado na Figura 1.

A manutenção da estrutura cristalina com alta porosidade, aliada às características geométricas das cavidades e aos efeitos entrópicos, são atrativos para a aplicação da UiO-67 e estruturas análogas no reconhecimento molecular, ${ }^{11}$ adsorção/dessorção de gases, ${ }^{12}$ catálise ${ }^{13}$ e funcionalização dos microambientes com compostos fotoquimicamente ativos, ${ }^{14}$ possibilitando a derivação de novos sensores, catalisadores e fotodispositivos.

No contexto da funcionalização de microambientes, a imobilização de complexos metálicos nas cavidades ou na superfície das MOFs permite que reações químicas ou fotoquímicas possam se dar nos sítios ativos das moléculas confinadas nos espaços interlamelares, aumentando a seletividade e eficiência dos processos reacionais ou catalíticos. ${ }^{15} \mathrm{Na}$ área fotofísica, espécies excitadas podem ser geradas com um tempo de vida (ou de emissão) bem maior, quando confinadas nas cavidades das matrizes. ${ }^{16}$ Essa abordagem amplia consideravelmente o espectro de utilização e de interesse dos sistemas, visto que os íons metálicos constituem centros de reatividade substitucional, redox ou fotoquímica e, quando colocados 

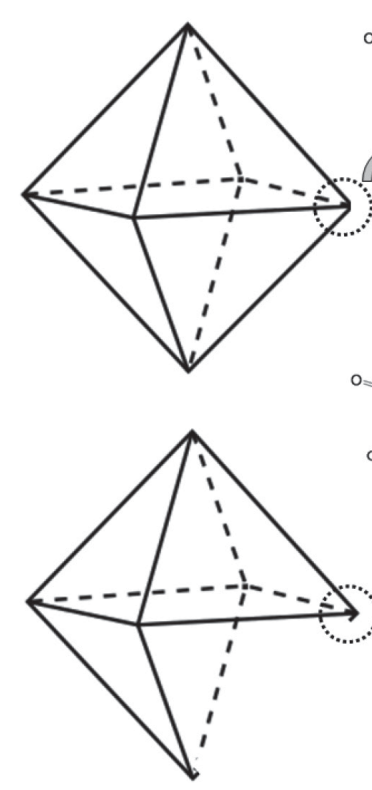

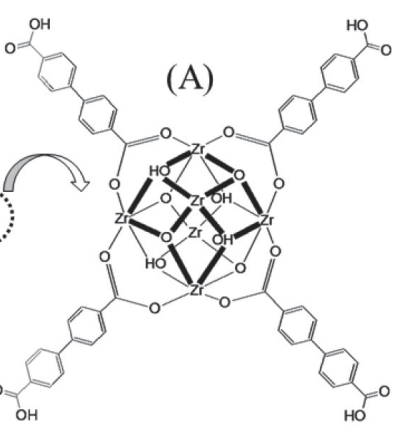

감

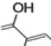

B)

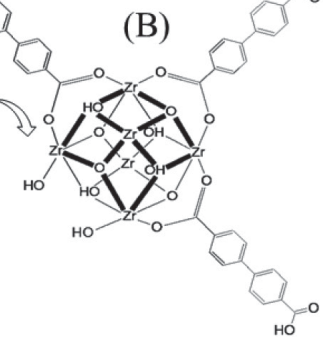

(C)

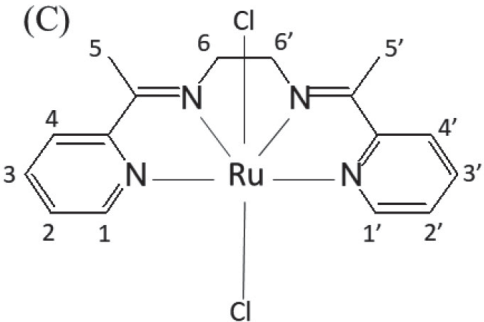

Figura 1. Representação do cluster $\left[\mathrm{Zr}_{6} \mathrm{O}_{4}(\mathrm{OH})_{4}\right]$ ligado a moléculas de ácido 4,4'-bisfenildicarboxílico (BPDC), sendo (A) estrutura da MOF UiO-67 sem defeito; (B) com defeito estrutural provocado pela vacância da molécula do ácido 4,4'-bisfenildicarboxílico; (C) do complexo de rutênio [RuCl 2 (ENAPY)] contendo a numeração para átomos de hidrogênio para auxiliar na interpretação do espectro de $R M N^{l} H$ (material suplementar)

em ambientes apropriados, proporcionam atividade catalítica e de sensoriamento analítico. ${ }^{17}$ Recentemente, Chen e colaboradores ${ }^{18}$ reportaram a aplicação de nanopartículas de UiO-67 modificadas com o íon complexo $\left[\mathrm{Ru}(\mathrm{bpy})_{3}\right]^{2+}$ para a detecção precoce de tumores utilizando diagnóstico por imagem fluorescente, que permite a visualização de células sem alterar sua morfologia ou integridade dos componentes celulares. Além disso, Chen e colaboradores ${ }^{18}$ mostraram que as nanopartículas de UiO-67 modificadas com o íon complexo de rutênio são fotossensíveis e induzem a morte células tumorais humanas do tipo A549 (câncer de pulmão) pela geração de moléculas de oxigênio singlete.

A combinação da estrutura das unidades metalo-orgânicas, em particular da MOF UiO-67, com as características espectroscópicas e eletroquímicas dos complexos de rutênio apresenta-se como uma opção para a detecção e determinação de moléculas gasosas de interesse ambiental. Contudo, antes de explorar a especificidade da matriz (MOF), somada à característica eletrônica do centro metálico, é necessário estudar as interações existentes entre o centro metálico e a cavidade da MOF. Assim, o objetivo desse trabalho é imobilizar o complexo $\left[\mathrm{RuCl}_{2}(\mathrm{ENAPY})\right]$, sendo ENAPY $=$ N,N'-bis(7-metil2-piridilmetileno)-1,2-diiminoetano, na estrutura metalo-orgânica UiO-67 e, por meio de técnicas espectroscópicas e eletroquímica, avaliar a influência da MOF nas características estrutural, eletrônica e redox do complexo de rutênio. As estruturas do complexo e da MOF UiO-67 estão representadas na Figura 1.

\section{PARTE EXPERIMENTAL}

Os reagentes cloreto de rutênio hidratado (Aldrich), 2-acetilpiridina (Aldrich), etilenodiamina (Aldrich), cloreto de zircônio (Merck), ácido 4,4'-bifenildicarboxílico (BPDC, Merck), etanol (Merck), acetonitrila (Merck), dimetilformamida (Merck) e diclorometano (Merck) foram de grau analítico e utilizados sem purificação prévia na síntese dos compostos. O complexo de rutênio e a MOF UiO-67 foram sintetizados conforme procedimentos descritos na literatura. ${ }^{8,19}$ Para o complexo de rutênio, o resultado de análise elementar é coerente com a fórmula $\left[\mathrm{RuCl}_{2} \mathrm{C}_{16} \mathrm{H}_{18} \mathrm{~N}_{4}\right]$ - experimental (calculado): $\mathrm{C}$ : 43,79 (43,84); H: 4,19 (4,15); N: 12,72 (12,78)\%.

\section{Modificação da MOF UiO-67 pelo complexo de rutênio}

Inicialmente foram preparadas as soluções: (A): $10 \mathrm{mg}$ do complexo de rutênio dissolvidos em $5 \mathrm{ml}$ de diclorometano; (B): $30 \mathrm{mg}$ da MOF UIO-67 adicionados a $5 \mathrm{ml}$ de diclorometano. A mistura foi submetida à agitação (agitador tipo vortex) por 5 minutos. Em seguida, misturou-se as soluções A e B, deixando em agitação mecânica por 3 dias. Posteriormente, centrifugou-se a mistura reacional por 15 minutos em $2000 \mathrm{rpm}$. O sólido obtido foi ressuspendido em $5 \mathrm{~mL}$ de $\mathrm{CH}_{2} \mathrm{Cl}_{2}$ e centrifugado por mais 15 minutos a $2000 \mathrm{rpm}$. Esse processo foi realizado três vezes. Por fim, o sólido foi ressuspendido em $5 \mathrm{~mL}$ de acetona e centrifugado (esse processo foi realizado duas vezes).

\section{Caracterização}

Espectro de $\mathrm{RMN}$ de ${ }^{1} \mathrm{H}$ do complexo de rutênio, dissolvido em $\mathrm{CDCl}_{3}$, foi obtido à temperatura ambiente em um espectrômetro Varian Inova operando a $300 \mathrm{MHz}$. Os teores de C, H, N foram determinados no equipamento Perkin Elmer CHN-2000. Os difratogramas de raios $\mathrm{X}$ foram obtidos em um difratômetro AXS D8 Advance Bruker, utilizando radiação $\mathrm{Cu}-\mathrm{K}_{\mathrm{a}}(\lambda=1,541 \AA)$ com ângulo de difração $(2 \theta)$ na faixa de 5 a $40^{\circ}$ em passo de $0,04^{\circ}$. Espectros de refletância difusa dos compostos sólidos foram obtidos em espectrofotômetro Lambda-1050 PerkinElmer. As análises de UV-Vis foram realizadas em soluções aquosas e acetonitrila em cubetas de quartzo e obtidas em um espectrofotômetro UV-VIS Cary 50 Scan Varian. Espectros FTIR foram obtidos em espectrômetro Nicolet iZ10, Thermo Fisher Scientific, em pastilha de $\mathrm{KBr}$ na região de 4000 a $400 \mathrm{~cm}^{-1}$. A composição dos elementos que constituem o sistema MOF-complexo de rutênio foi analisada por espectroscopia fotoeletrônica de raios X, XPS, utilizando equipamento VG Microtech, anodo de $\mathrm{Mg}$ (risca $\mathrm{K} \alpha$ ), analisador semi-hemisférico de $150 \mathrm{~mm}$ e 9 channeltrons, pressão de trabalho de $1 \times 10^{-9} \mathrm{mBar}, \mathrm{CAE}=50 \mathrm{eV}$ e resolução energética de 0,80 eV. Voltamogramas cíclicos foram obtidos em potenciostato Metrohm Autolab, modelo PGSTAT101, utilizando solução de acetonitrila contendo $0,10 \mathrm{~mol} \mathrm{~L}^{-1}$ de perclorato de lítio como eletrólito suporte. A célula eletroquímica foi composta por: eletrodo de trabalho: platina; 
eletrodo de referência: $\mathrm{Ag} / \mathrm{AgNO}_{3} 0,01 \mathrm{~mol} \mathrm{~L}^{-1}$ e perclorato de lítio $0,10 \mathrm{~mol} \mathrm{~L}^{-1}$; eletrodo auxiliar: fio de platina.

\section{RESULTADOS E DISCUSSÃO}

\section{Caracterização dos precursores}

Ressonância Magnética Nuclear de ${ }^{1} \mathrm{H}$

A composição da esfera de coordenação ao redor do íon rutênio(II) foi inferida pela análise do espectro de $\mathrm{RMN}$ de ${ }^{1} \mathrm{H}$ do complexo dissolvido em clorofórmio deuterado (Figura 1S). No espectro foram observados dois sinais dubletos $[\delta=8,65 \mathrm{ppm}$; $\delta=8,04 \mathrm{ppm}]$ e dois sinais tripletos $[\delta=7,84 \mathrm{ppm} ; \delta=7,48 \mathrm{ppm}]$, que foram atribuídos aos prótons aromáticos dos anéis piridínicos do ligante ENAPY coordenado ao centro metálico. Os prótons dos grupos $\mathrm{CH}_{3}$ foram associados ao sinal de ressonância com valor de deslocamento químico igual a 2,75 ppm. Verificou-se, também, um singleto em $\delta=2,60 \mathrm{ppm}$ que foi atribuído aos prótons dos grupos $\mathrm{CH}_{2}$. A partir dos valores de integração dos sinais de ressonância, determinou-se a relação estequiométrica entre o ligante ENAPY e o centro metálico que, neste caso, foi de 1:1.

O padrão dos sinais de ressonância observado é coerente com a existência de um composto simétrico e, dentre os isômeros possíveis para esse sistema, ${ }^{20}$ pode-se concluir que o ligante ENAPY coordenou-se ao centro metálico através dos quatro átomos de nitrogênio disponíveis: dois dos grupos imina e dois dos anéis piridínicos. Essas ligações perfazem as interações no plano equatorial do complexo, que tem os dois anéis piridínicos e as duas iminas em posições vicinais entre si. As posições axiais do complexo são ocupadas por íons cloreto. As atribuições foram corroboradas por dados cristalográficos descritos na literatura. ${ }^{19}$

\section{Difratometria de raios $X$ : método do pó}

A difratometria de raios $X$ foi utilizada para a identificação e caracterização do sólido obtido da reação de cloreto de zircônio e ácido 4,4'-bisfenildicarboxilico (BPDC). O difratograma do sólido, Figura $2 \mathrm{~S}$, foi comparado com os dados descritos na literatura ${ }^{21} \mathrm{e}$ o padrão de difração obtido foi característico da estrutura metalo-orgânica UiO-67. A estrutura da MOF é mantida pelas ligações de ponte do zircônio com o ácido 4,4'-bisfenildicarboxílico, pela formação de ligações com o oxigênio central na unidade $\mathrm{Zr}_{3} \mathrm{O}$ (classificadas como $\mu_{3}-\mathrm{O}$ ), além das ligações metal-OH localizadas no triangulo $\mathrm{Zr}_{3}$ (classificadas como $\mu_{3}-\mathrm{OH}$ ). A estrutura desse cluster é estável, resultando em um sólido cristalino, de estrutura cúbica de face centrada e grupo espacial Fm3m, cuja organização do reticulo cristalino resulta em cavidades octaédricas de diâmetro $\sim 20 \AA$ e interstícios tetraédricos de diâmetro de $\sim 12 \AA{ }^{8}$

\section{Caracterização do sistema MOF UiO-67/[ $\mathrm{RuCl}_{2}$ (ENAPY)]}

O indicativo da imobilização do complexo de rutênio na MOF UiO-67 foi dado pela mudança de coloração da UiO-67. A MOF inicialmente branca adquiriu coloração azul. Dois dias após a síntese, o sistema UiO-67/complexo de cor azul mudou de coloração, passando a apresentar a cor vermelha. Essa alteração de coloração é justificada pelo comportamento do complexo de rutênio na presença de água. A reação do cloreto de rutênio com o ligante imínico ENAPY, em etanol e presença de cloreto de lítio, leva à formação do complexo $\left[\mathrm{RuCl}_{2}(\mathrm{ENAPY})\right]$ que dissolvido em solventes orgânicos gera soluções de cor azul. Na presença de água, ocorre a troca dos ligantes axiais (de íons $\mathrm{Cl}^{-}$por moléculas de água) levando à formação do aquocomplexo de rutênio $\left[\mathrm{Ru}(\mathrm{ENAPY})\left(\mathrm{H}_{2} \mathrm{O}\right)_{2}\right] \mathrm{Cl}_{2}$, de coloração vermelha, como pode ser observado na Figura 2. O processo de aquação do complexo de rutênio já está descrito na literatura ${ }^{22} \mathrm{e}$, por isso, optamos por não reproduzi-lo aqui. Dessa forma, os dados espectroscópicos e eletroquímicos apresentados a seguir correspondem ao sistema UiO-67/[Ru(ENAPY) $\left.\left(\mathrm{H}_{2} \mathrm{O}\right)_{2}\right] \mathrm{Cl}_{2}$.

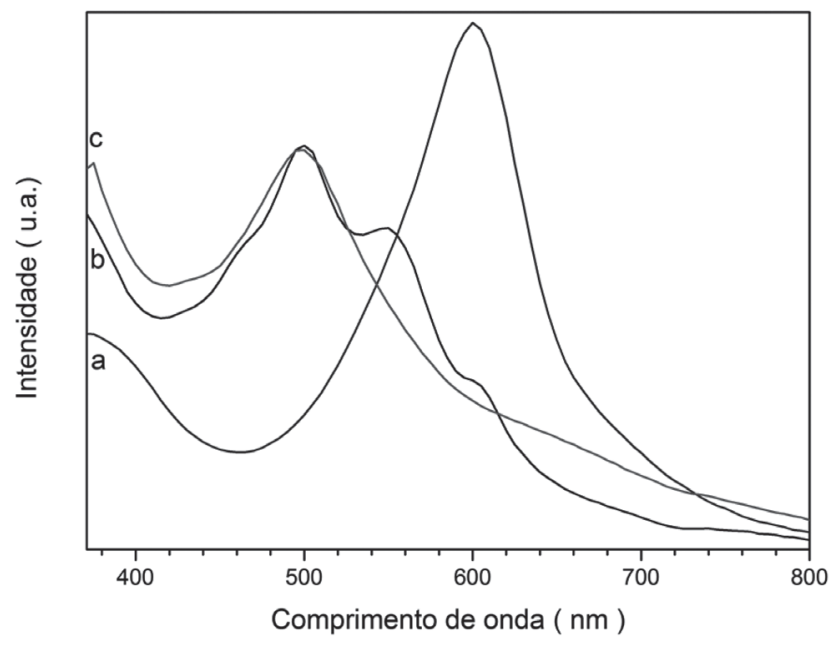

Figura 2. Espectros eletrônicos do complexo de rutênio em: (a) acetonitrila $\left\{\left[R u C l_{2}(E N A P Y)\right]\right\} ;(\boldsymbol{b})$ acetonitrila contendo $5 \%$ v/v de água destilada após 5 minutos $\left\{\left[\mathrm{RuCl}\left(\mathrm{H}_{2} \mathrm{O}\right)(\text { ENAPY })\right]^{+}\right.$intermediário, não isolado\}; (c) acetonitrila contendo $5 \% v / v$ de água destilada após 20 minutos $\left\{\left[\mathrm{Ru}(\mathrm{ENAPY})\left(\mathrm{H}_{2} \mathrm{O}\right)_{2}\right]^{2+}\right\}$, à temperatura ambiente

Além disso, a análise de XPS comprovou a inserção do complexo na unidade MOF UIO-67, que é constituída de 1,0\% de Zircônio; $68,7 \%$ de Carbono; $3,0 \%$ de Oxigênio; $26,8 \%$ de Nitrogênio e 0,4\% de Rutênio em massa do sistema. Na Figura 3 o pico em $400 \mathrm{eV}$ foi atribuído à transição de elétrons envolvendo orbitais $1 \mathrm{~s}$ e 2s dos átomos de nitrogênio, bem como o pico em $462 \mathrm{eV}$ foi associado à transição eletrônica centrada no rutênio $\left(3 \mathrm{p}^{2 / 3} \AA 4 \mathrm{~d}^{3 / 2}\left(\right.\right.$ ou $\left.\left.4 \mathrm{~d}^{5 / 2}\right)\right) .^{23}$

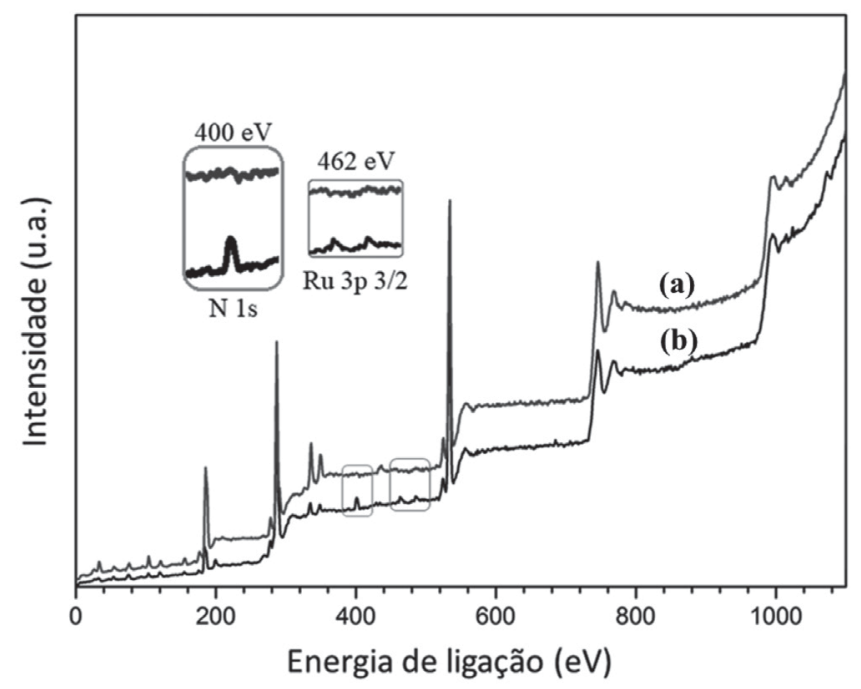

Figura 3. Espectros de XPS da MOF UiO-67 (a), e da MOF UiO-67 modificada com complexo de rutênio (b). Em destaque os sinais referentes as transições eletrônicas envolvendo orbitais $1 s$ do $\mathrm{Ne} 3 p^{2 / 3}$ do $\mathrm{Ru}(\mathrm{II})$

Espectros eletrônicos na região do UV-Visível

$\mathrm{O}$ espectro eletrônico do complexo imobilizado na MOF UiO-67 obtido por refletância difusa, Figura $4 b$, apresentou uma banda em $\lambda=520 \mathrm{~nm}$ e um ombro em $\lambda=380 \mathrm{~nm}$ atribuídos aos processos de transferência de carga metal 
- ligante $\left\{\right.$ MLCT, $\left.\mathrm{Ru}\left(\mathrm{t}_{2 \mathrm{~g}}{ }^{6}\right) \rightarrow \pi^{*}(\mathrm{ENAPY})\right\} .{ }^{22,24}$ Comparando-se os espectros do complexo em solução (Figura 4c) e imobilizado na MOF (Figura 4b), nota-se um deslocamento batocrômico da banda $\operatorname{MLCT}\left(\lambda_{\text {solução }}=500 \mathrm{~nm} ; \lambda_{\text {imobilizado }}=520 \mathrm{~nm}\right)$. Este deslocamento pode ser justificado pelas interações do complexo de rutênio com as cavidades da matriz via ligações de hidrogênio das moléculas de água coordenadas axialmente ao centro metálico com as hidroxilas presentes no cluster de zircônio $\left\{\mathrm{Zr}-\mu_{3}-\mathrm{OH} \cdots \cdot \mathrm{H}_{2} \mathrm{O}-\mathrm{Ru}^{\mathrm{II}}-\mathrm{H}_{2} \mathrm{O} \cdots \cdot \mathrm{OH}-\mu_{3}-\mathrm{Zr}\right\}$. As ligações de hidrogênio aproximam a base de Schiff coordenada ao rutênio dos ligantes 4,4'-bisfenildicarboxilicos (BPDC) que conectam os clusters de zircônio, favorecendo as interações das nuvens eletrônicas dos anéis aromáticos desses ligantes. As interações $\pi-\pi$ dos ligantes ENAPY BPDC, por efeito indutivo de corrente de anel, diminuem a densidade eletrônica ao redor do centro metálico, estabilizando os níveis $\mathrm{d} \pi$ do rutênio(II) e, consequentemente, deslocando a banda MLCT do complexo de rutênio.

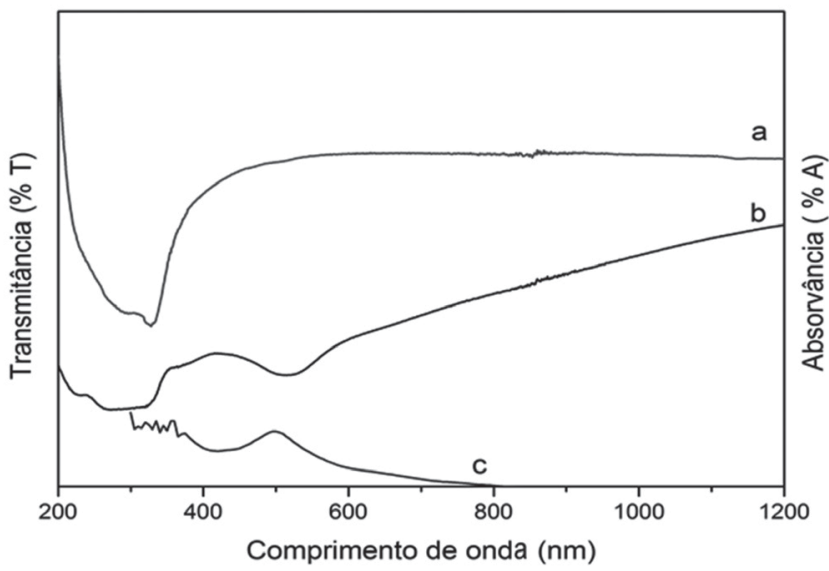

Figura 4. Espectros eletrônicos na região do UV-Visível dos compostos: (a) MOF UiO-67 (modo refletância difusa); (b) MOF UiO-67 modificada com complexo de rutênio (modo refletância difusa); (c) complexo de rutênio em solução aquosa

\section{Voltametria Cíclica}

A matriz UiO-67 não apresentou processos redox associados aos clusters de zircônio, tampouco às moléculas de solventes inclusas nas cavidades da estrutura metalo-orgânica, como mostrado na Figura 5a, o que está de acordo com o comportamento eletroquímico dessa classe de MOF conforme dados da literatura. ${ }^{25}$

No voltamograma cíclico do complexo de rutênio, em acetonitrila - Figura 5b, verificou-se um par de ondas reversível e monoeletrônico com valor de $\mathrm{E}_{1 / 2}=-0,05 \mathrm{~V}$ (vs $\mathrm{Ag} / \mathrm{Ag}^{+}$) atribuído ao processo redox $\mathrm{Ru}^{\mathrm{II}}-\mathrm{Ru}^{\mathrm{III}}$. Assim como nos complexos polipiridínicos de rutênio ${ }^{26} \mathrm{O}$ processo de oxidação do $\left[\mathrm{RuCl}_{2}\right.$ (ENAPY)] é centrado no metal e leva à formação da espécie $\left[\mathrm{Ru}^{\mathrm{II}} \mathrm{Cl}_{2}(\mathrm{ENAPY})\right]^{+1}$, de configuração $\mathrm{d} \pi\left(\mathrm{t}_{2 \mathrm{~g}}{ }^{5}\right)$. Ao adicionar água à solução do complexo dissolvido em acetonitrila ( $0,5 \mathrm{~mL}$ de água em $5 \mathrm{~mL}$ de acetonitrila), Figura $5 \mathrm{c}$, verificou-se dois pares de ondas reversíveis com valores de $\mathrm{E}_{1 / 2}=-0,05 \mathrm{~V} \mathrm{e} \mathrm{E}_{1 / 2}=+0,45 \mathrm{~V}$ (vs $\mathrm{Ag} / \mathrm{Ag}^{+}$) atribuídos aos processos redox $\mathrm{Ru}^{\mathrm{II}}-\mathrm{Ru}^{\mathrm{III}}$ das espécies $\left[\mathrm{RuCl}_{2}(\mathrm{ENAPY})\right]$ e $\left[\mathrm{Ru}\left(\mathrm{H}_{2} \mathrm{O}\right)_{2}(\mathrm{ENAPY})\right]^{2+}$, respectivamente.

A substituição dos ligantes axiais (de $\mathrm{Cl}^{-}$por $\mathrm{H}_{2} \mathrm{O}$ ) fez com que o potencial de oxidação do centro metálico deslocasse em aproximadamente $400 \mathrm{mV}$ no sentido positivo, que pode ser justificado pelo acréscimo de carga positiva sobre o complexo em função da troca de um ligante $\sigma / \pi$-doador (íon cloreto, classificado como ligante de campo fraco) por outro ligante com caráter $\sigma$-doador (molécula de água, classificada como ligante de campo moderado).

No voltamograma cíclico do sistema UiO-67/complexo de rutênio, Figura 5d, observou-se apenas um par de ondas reversível com valor de $\mathrm{E}_{1 / 2}=0,25 \mathrm{~V}\left(v s \mathrm{Ag} / \mathrm{Ag}^{+}\right)$atribuído à oxidação/redução do centro metálico $\left\{\mathrm{Ru}^{2+}\left(\mathrm{t}_{2 \mathrm{~g}}{ }^{6}\right) \leftrightarrows \mathrm{Ru}^{3+}\left(\mathrm{t}_{2 \mathrm{~g}}{ }^{5}\right)\right\}$.

Como mencionado anteriormente, o complexo de rutênio incorporado na matriz UiO-67 é o $\left[\mathrm{Ru}\left(\mathrm{H}_{2} \mathrm{O}\right)_{2}(\text { ENAPY) }]^{2+}\right.$, haja vista a tendência de aquação do complexo precursor. Contudo, quando comparamos os valores de potencial de oxidação do aquocomplexo em solução $\left(E_{1 / 2}=0,45 \mathrm{~V}\right)$ e imobilizado $\left(\mathrm{E}_{1 / 2}=0,25 \mathrm{~V}\right)$ notamos um deslocamento de $200 \mathrm{mV}$, o que é coerente com o comportamento espectroscópico e reflete a estabilização dos níveis $\mathrm{d} \pi$ do metal no aquocomplexo induzida pelas interações do complexo de rutênio com as cavidades da matriz via ligações de hidrogênio das moléculas de água coordenadas axialmente ao centro metálico com as hidroxilas presentes no cluster de zircônio $\left\{\mathrm{Zr}-\mu_{3}-\mathrm{OH} \cdots \cdot \mathrm{H}_{2} \mathrm{O}-\mathrm{Ru}^{\mathrm{II}}-\mathrm{H}_{2} \mathrm{O} \cdots \cdot \mathrm{OH}-\mu_{3}-\mathrm{Zr}\right\}$.

\section{Espectros vibracionais na região do Infravermelho (FTIR)}

Como mencionado, a UiO-67 é constituída por clusters octaédricos de $\left[\mathrm{Zr}_{6} \mathrm{O}_{4}(\mathrm{OH})_{4}\right]$ ligados a moléculas de ácido 4,4'-bisfenildicarboxílico, formando uma estrutura supramolecular organizada, simétrica e tridimensional com cavidades octaédricas e tetraédricas. ${ }^{8}$ O cluster de zircônio é estabilizado por grupos carboxílicos na conformação eclipsada (syn-syn), por átomos de oxigênio e grupos hidroxilas ligados aos íons de zircônio(IV), formando as interações do tipo $\mu_{3}-\mathrm{O}$ e e $\mu_{3}-\mathrm{OH}$, como ilustrado na Figura 1.

O espectro vibracional da MOF UiO-67, Figura 6b, é caracterizado por uma banda intensa e alargada na região de $3500 \mathrm{~cm}^{-1}$ atribuída aos estiramentos assimétricos das ligações $\mathrm{O}-\mathrm{H}$ de moléculas de água adsorvidas na MOF somados aos estiramentos assimétricos dos fragmentos hidroxilas $\mu_{3}-\mathrm{OH}$ da estrutura metal-orgânica. Em $1670 \mathrm{~cm}^{-1}$ e 1630 $\mathrm{cm}^{-1}$ foram observadas bandas relativamente intensas atribuídas aos estiramentos assimétricos em fase e fora de fase dos grupos $\mathrm{COO}^{-}$dos ácidos 4,4'-bisfenildicarboxílicos coordenados aos centros metálicos, respectivamente. A presença dos ácidos 4,4'-bisfenildicarboxílicos ligados aos íons de $\mathrm{Zr}(\mathrm{IV})$ também foi corroborada pelas bandas observadas em $1540 \mathrm{~cm}^{-1}$ associada ao estiramento assimétrico das ligações $\mathrm{C}=\mathrm{C}$ dos anéis aromáticos $\left(\mathrm{v}_{\mathrm{as}} \mathrm{C}=\mathrm{C}_{\text {anel }}\right), 1425 \mathrm{~cm}^{-1}$ atribuída ao estiramento simétrico dos fragmentos carboxílicos $\left(v_{\mathrm{s}} \mathrm{COO}\right), 1350 \mathrm{~cm}^{-1}$ atribuída ao estiramento simétrico das ligações $\mathrm{C}=\mathrm{C}$ dos anéis aromáticos e 735 $\mathrm{cm}^{-1}$ associada às deformações angulares das ligações $\mathrm{C}=\mathrm{C}$ e COODuas bandas de intensidade relativamente fraca foram observadas em $620 \mathrm{~cm}^{-1}$ e $530 \mathrm{~cm}^{-1}$ atribuídas aos estiramentos assimétrico e simétrico, respectivamente, das ligações $\mathrm{Zr}-\mathrm{OC}=\mathrm{O}$. As bandas observadas em $680 \mathrm{~cm}^{-1} \mathrm{e} 440 \mathrm{~cm}^{-1}$ foram associadas aos estiramentos dos átomos de oxigênio e dos grupos hidroxilas ligados aos íons de zircônio(IV), formando as interações do tipo $\mu_{3}-\mathrm{O}$ e $\mu_{3}-\mathrm{OH}$, respectivamente.

A imobilização do complexo de rutênio na MOF UiO-67 não alterou significativamente os modos vibracionais na unidade metalo-orgânica, como podemos observar no espectro vibracional do sistema UiO-67/complexo de rutênio, Figura 6a. Nesse caso, observamos um conjunto de bandas de intensidade relativamente fraca na região de $2900 \mathrm{~cm}^{-1}$ atribuído aos estiramentos assimétricos das ligações C-H dos fragmentos $\mathrm{CH}_{2}$ e $\mathrm{CH}_{3}$ da base de Schiff coordenada ao rutênio. Verificamos também a ausência da banda em $1730 \mathrm{~cm}^{-1}$ (presente no espectro vibracional do complexo puro, Figura 6c) característica da deformação fora do plano do anel piridínico da base de Schiff. Além disso, notamos um deslocamento de $18 \mathrm{~cm}^{-1}$ da banda associada ao estiramento do fragmento $\mu_{3}-\mathrm{OH}$ da estrutura metalo-orgânica após a imobilização do complexo de rutênio $\left(v_{\mu 3-\mathrm{OH}}=440 \mathrm{~cm}^{-1}\right.$ para UiO-67; $v_{\mu 3-\mathrm{OH}}=458 \mathrm{~cm}^{-1}$ para UiO-67/complexo).

A partir dessas observações podemos inferir que interação do complexo de rutênio com a UiO-67 ocorreu próxima aos fragmentos $\mu_{3}-\mathrm{OH}$, cuja localização está destacada na Figura 1. As dimensões 

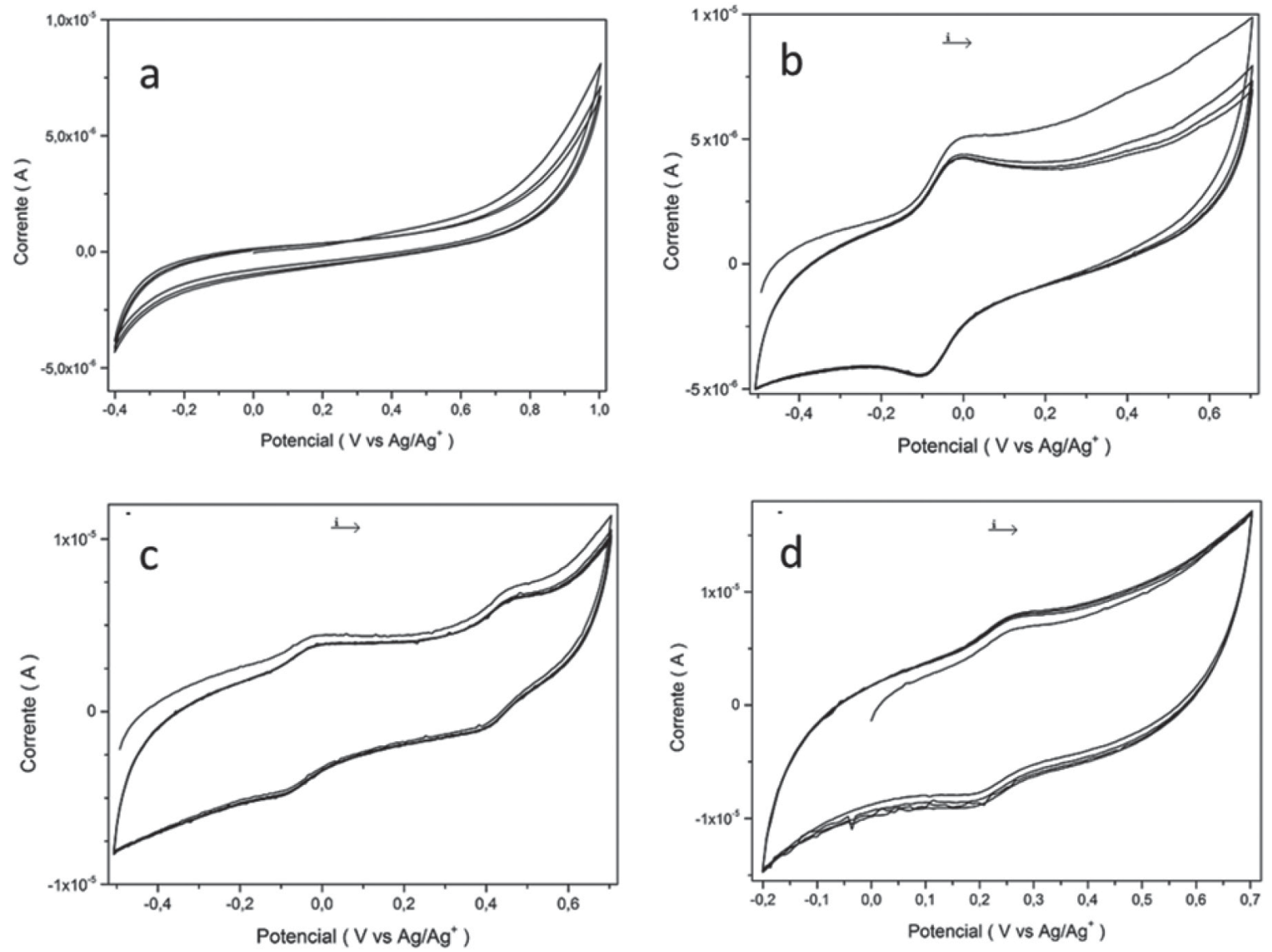

Figura 5. Voltamogramas cíclicos de: (a) MOF UiO-67; (b) complexo de rutênio em acetonitrila; (c) complexo de rutênio em acetonitrila contento água destilada; (d) MOF UiO-67 modificada com complexo de rutênio. Solvente acetonitrila contendo 0,10 mol L-1 de $\mathrm{LiClO}_{4}$, eletrodo de trabalho: platina; eletrodo de referência: $\mathrm{Ag} / \mathrm{AgNO}_{3} 0,01 \mathrm{~mol} \mathrm{~L}^{-1}+\mathrm{LiClO}_{4} 0,10 \mathrm{~mol} \mathrm{~L}^{-1}$; eletrodo auxiliar: fio de platina, sentido de varredura anódico, velocidade de varredura $100 \mathrm{mV} \mathrm{s}^{-1}$

desses microambientes tendem a dificultar os movimentos de deformação fora do plano dos anéis piridínicos da base de Schiff $\left(v_{\text {py-oop }}\right)$, o que justifica o desaparecimento da banda atribuída a esse modo vibracional. Finalmente, os dados vibracionais aliados ao difratograma de raios-X de pó indicaram que as características estruturais da MOF não foram alteradas pela presença do complexo de rutênios nos microambientes da UiO-67.

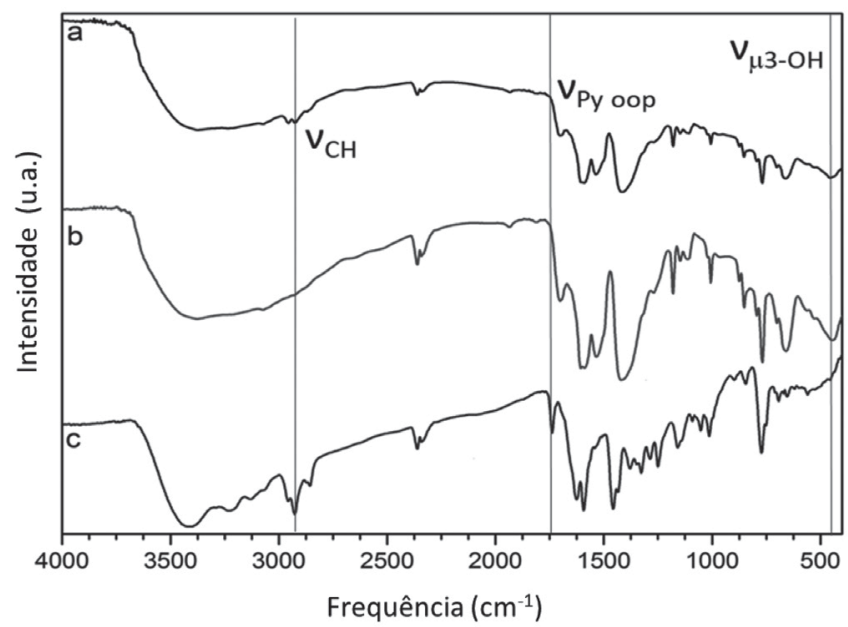

Figura 6. Espectros vibracionais na região do infravermelho dos compostos: (a) MOF UiO-67 modificada com complexo de rutênio; (b) MOF UiO-67; (c) complexo de rutênio, obtidos em pastilha de $\mathrm{KBr}$

\section{Difratometria de raios $X$ : método do pó}

No difratograma do complexo de rutênio, Figura $7 \mathrm{c}$, verificou-se a ausência de picos de difração, revelando a característica amorfa desse derivado metálico. Para a matriz UiO-67 foram obtidos dois difratogramas: o primeiro logo após a síntese da MOF, Figura 7b', e o segundo após o processo de secagem da amostra, Figura 7b. O difratograma obtido da MOF UiO-67 logo após a síntese apresentou apenas dois picos de difração com valores de $2 \theta=5,7^{\circ}$ e $6,6^{\circ}$ correspondentes aos planos [111] e [200] da estrutura metalo-orgânica, revelando baixo grau de cristalinidade do pó. Por outro lado, o difratograma da MOF UiO-67 obtido após o processo de secagem apresentou picos de difração melhores definidos, indicando elevado grau de cristalinidade da amostra. A atribuição dos picos de difração do pó da matriz UiO-67 está apresentada na Figura 2S, por isso optamos por não reproduzir nessa etapa.

A imobilização do complexo de rutênio na UiO-67 não alterou significativamente os picos de difração da MOF, como podemos observar no difratograma da Figura 7a, mostrando que o arranjo cristalino da MOF é preservado. No entanto, a intensidade relativa dos picos de difração com valores de $2 \theta=9,2^{\circ}, 13,5^{\circ}$ e $20^{\circ}$ foram alteradas após a inclusão do complexo na matriz (intensidade de pico antes da inclusão: $\mathrm{I}_{9,2}=30,4 ; \mathrm{I}_{13,5}=24,3 ; \mathrm{I}_{20}=26,7$; intensidade de pico após a inclusão: $\mathrm{I}_{9,2}=44,7 ; \mathrm{I}_{13,5}=42,6 ; \mathrm{I}_{20}=41,3$ ). Essa alteração de intensidade dos picos sugere que o complexo de rutênio está inserido na MOF e promove uma readequação dos microambientes da estrutura cristalina correspondentes aos planos [220], [400] e [442]. 


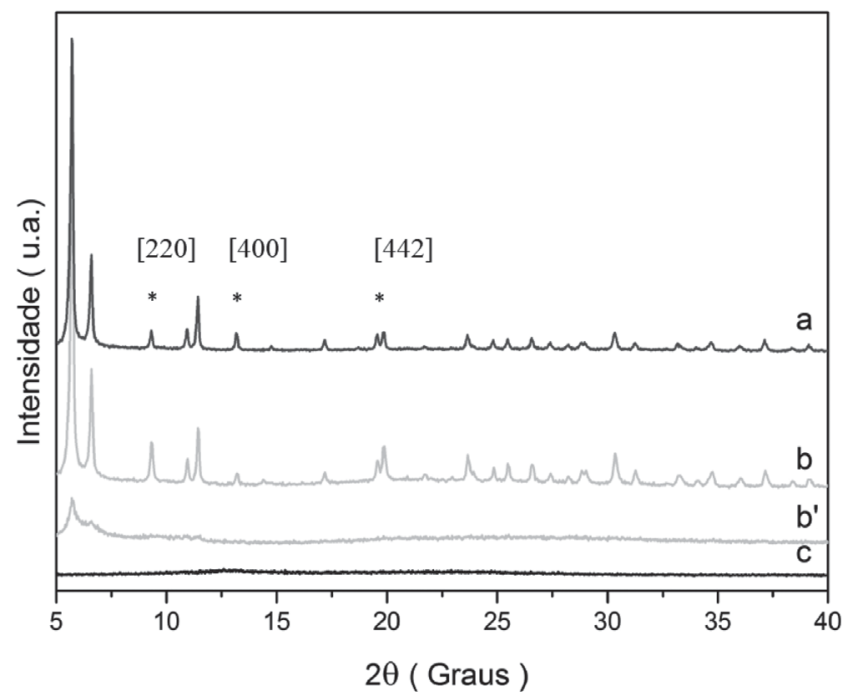

Figura 7. Difratogramas de raio-X método do pó dos compostos: (a) MOF UiO-67 modificada com complexo de rutênio; (b) MOF UiO-67 após etapa de secagem; (b')MOF UiO-67 antes da etapa de secagem; (c) complexo de rutênio

\section{Método Brunauer-Emmett-Teller}

O método BET forneceu as áreas superficiais dos sólidos UiO-67 e UiO-67 modificada com o complexo de rutênio. A MOF UIO-67 apresentou uma área superficial de aproximadamente $1900 \mathrm{~m}^{2}$ por grama de composto, o que é coerente com os dados descritos na literatura para os derivados UiO. ${ }^{8-10}$ Já a MOF modificada com o complexo de rutênio apresentou valor de aproximadamente $1400 \mathrm{~m}^{2}$ por grama de composto. Com a inserção do complexo na MOF houve um decréscimo de área disponível na estrutura cristalina e porosa da UiO-67, revelando que o derivado de rutênio está inserido nos microambientes da matriz e compete com as moléculas de $\mathrm{N}_{2}$ pelo espaço disponível na amostra de UiO-67.

\section{CONCLUSÃO}

A imobilização do complexo de rutênio com a unidade MOF UiO-67 ocorreu de forma espontânea e foi direcionada por ligações de hidrogênio das moléculas de água coordenadas axialmente ao centro metálico com as hidroxilas presentes no cluster de zircônio. As ligações intermoleculares aproximaram a base de Schiff coordenada ao rutênio dos ligantes 4,4'-bisfenildicarboxilicos (BPDC) que conectam os clusters de zircônio, favorecendo as interações das nuvens eletrônicas dos anéis aromáticos desses ligantes que, por efeito indutivo de corrente de anel, diminuiram a densidade eletrônica ao redor do centro metálico, estabilizando os níveis $\mathrm{d} \pi$ do rutênio(II). A imobilização do complexo de rutênio na estrutura da UiO-67 promoveu uma readequação dos microambientes da estrutura cristalina, a qual diminuiu a capacidade de absorção e armazenamento de gás nos microambientes da MOF.

\section{MATERIAL SUPLEMENTAR}

Estão disponíveis em http://quimicanova.sbq.org.br, com acesso livre, o espectro de $\mathrm{RMN}{ }^{1} \mathrm{H}$ do complexo $\left[\mathrm{RuCl}_{2}(\mathrm{~L})\right]$ obtido em clorofórmio deuterado (Figura $1 \mathrm{~S}$ ) e o difratograma de raios-X, método do pó, do composto precursor UiO-67 contendo os índices de Miller para planos da estrutura cúbica da MOF (Figura 2S).

\section{AGRADECIMENTOS}

Os autores agradecem à Fundação Araucária/Paraná e ao CNPq pelo suporte financeiro na execução do projeto de grupo.

\section{REFERÊNCIAS}

1. Batten, S. R.; Champness, N. R.; Chen, X.-M.; Garcia-Martinez, J.; Kitagawa, S.; Öhrstrom, L.; O’Keeffe, M.; Suh, M. P.; Reedijk, J.; Pure Appl. Chem. 2013, 85, 1715; Oar-Arteta, L.; Wezendonk, T.; Sun, X.; Kapteijn, F.; Gascon, J.; Mater. Chem. Front. 2017, 1, 1709; Semino, R.; Moreton, J. C.; Ramsahye, N. A.; Cohen, S. M.; Maurin, G.; Chem. Sci. (2018), doi.: 10.1039/c7sc04152g.

2. Kaskel, S.; The Chemistry of Metal Organic Frameworks: Synthesis, Characterization, and Applications, vol. 1, $1^{\text {th }}$ ed., Wiley: New York, 2016.

3. Leong, W. L.; Vittal, J. J.; Chem. Rev. 2011, 111, 688.

4. Sinelshchikova, A. A.; Nefedov, S. E.; Enakieva, Y. Y.; Gorbunova, Y. G.; Tsivadze, A. Y.; Kadish, K. M.; Chen, P.; Bessmertnykh-Lemeune, A.; Stern, C.; Guikard, R.; Inorg. Chem. 2013, 52, 999.

5. Easun, T. L.; Jia, J.; Calladine, J. A.; Blackmore, D. L.; Stapleton, C. S.; Vuong, K. Q.; Champness, N. R.; George, M. W.; Inorg. Chem. 2014, 53, 2606; Tian, J.; Xu, Z.-Y.; Zhang, D.-W.; Wang, H.; Xie, S.-H.; Xu, D.-W.; Ren, Y.-H.; Wang, H.; Liu, Y.; Li, Z.-T.; Nature Commun. 2016, 7, 11580

6. Yap, M. H.; Fow, K. L.; Chen, G. Z.; Green Energy Environ. 2017, 2 , 218.

7. Cavka, J. H.; Jakobsen, S.; Olsbye, U.; Guillou, N.; Lamberti, C.; Bordiga, S.; Lillerud, K. P.; J. Am. Chem. Soc. 2008, 130, 13850.

8. Katz, M. J.; Brown, Z. J.; Colon, Y. J.; Siu, P. W.; Scheidt, K. A.; Snurr, R. Q.; Hupp, J. T.; Farha, O. K.; Chem. Commun. 2013, 49, 9449.

9. Lawrence, M. C.; Schneider, C.; Katz, M. J.; Chem. Commun. 2016, 52, 4971.

10. Gutov, O. V.; Hevia, M. G.; Escudero-Adan, E. C.; Shafir, A.; Inorg. Chem. 2015, 54, 8396.

11. Dalapati, R.; Biswas, S.; Sens. Actuators, B 2017, 239, 759.

12. Hu, J. B.; Liu, Y.; Liu, J.; Gu, C.K.; Wu, D. W.; Microporous Mesoporous Mater. 2018, 256, 25; Ahmad, M. Z.; Navarro, M.; Lhotka, M.; Zornoza, B.; Tellez, C.; Fila, V.; Coronas, J.; Sep. Purif. Technol. 2018, $192,465$.

13. Liang, H. X.; Jiao, X. L.; Li, C.; Chen, D. R.; J. Mater. Chem. A 2018, 6, 334. Shaabani, A.; Mohammadian, R.; Hooshmand, S. E.; Hashemzadeh, A.; Amini, M. M.; Chem. Select 2017, 2, 11906.

14. Larsen, R. W.; Mayers, J. M.; Wajtas, L.; Dalton Trans. 2017, 46, 12711; Zhao, J.; Wang, Q.; Sun, C. Y.; Zheng, T. T.; Yan, L. K.; Li, M. T.; Shao, K. Z.; Wang, X. L.; Su, Z. M.; J. Mater. Chem. A 2017, 5, 12498. Larsen, R.W.; Wojtas, L.; J. Solid State Chem. 2017, 247, 77; Guan, B. Y.; Yu, X. Y.; Wu, H. B.; Lou, X. W.; Adv. Mater. 2017, 29, 1703614; Downes, C. A.; Marinescu, S. C.; ChemSusChem 2017, 10, 4374; Chaudhari, A. K.; Ryder, M. R.; Tan, J.-C.; Nanoscale 2016, 8, 6851.

15. Takashima, Y.; Fukuhara, Y.; Sato, Y.; Tsuruoka, T.; Akamatsu, K.; Eur. J. Inorg. Chem. 2017, 45, 5344; Shu, X.; Yu, Y.; Jiang, Y.; Luan, Y.; Ramella, D.; Appl. Organomet. Chem. 2017, 31, 3862; Pourkhosravani, M.; Dehghanpour, S.; Farzaneh, F.; Sohrabi, S.; React. Kinet., Mech. Catal. 2017, 122, 961; Tang, L.; Shi, J. F.; Wang, X.L.; Zhang, S. H.; Wu, H.; Sun, H. F.; Jiang, Z. Y.; Nanotechnology 2017, 28, 275601.

16. Zhang, W. X.; Li, B.; Ma, H. P.; Zhang, L. M.; Guan, Y. L.; Zhang, Y. H.; Zhang, X. D.; Jing, P. T.; Yue, S. M.; ACS Appl. Mater. Interfaces 2017, 8, 21465; Chen, R.; Zhang, J.; Chelora, J.; Xiong, Y.; Kershaw, S. V.; Li, K. F.; Lo, P. K.; Cheah, K. W.; Rogach, A. L.; Zapien, J. A.; ACS Appl. Mater. Interfaces 2017, 9, 5699; Bennett, T. D.; Cheetham, A. K.; Acc. Chem. Res. 2014, 47, 1555.

17. Rogge, S. M. J.; Bavykina, A.; Hajek, J.; Garcia, H.; Olivos-Suarez, A. I.; Sepulveda-Escribano, A.; Vimont, A.; Clet, G.; Bazin, P.; Kapteijn, F.; Daturi, M.; Ramos-Fernandez, E. V.; Llabrés-Xamena, F. X.; van Speybroeck, V.; Gascom, J.; Chem. Soc. Rev. 2017, 46, 3134; Campbell, M. G.; Dincã, M.; Sensors 2017, 17, 1108; Lin, R.-B.; Liu, S.-Y.; Ye, J.-W.; Li, X.-Y.; Zhang, J.-P.; Adv. Sci. 2016, 3, 1500434. 
18. Chen, R.; Zhang, J.; Chelora, J.; Xiong, Y.; Kershaw, S. V.; Li, K. F.; Lo, P.-K.; Cheah, K. W.; Rogach, A. L.; Zapien, J. A.; Lee, C.-S.; ACS Appl. Mater. Interfaces 2017, 9, 5699.

19. Pal, S.; Pal, S.; Polyhedron 2003, 22, 867.

20. Da Silva, A. P.; Gauze, G. F.; Matias, T. A.; de Souza, V. R.; Rozada, T. C.; Basso, E.; Tetrahedron Lett. 2011, 52, 5043.

21. David, J.; Trolliard, G.; Volkringer, C.; Loiseau, T.; Maitre A.; RSC Adv. 2015, 5, 51650 .

22. de Souza, V. R.; Nunes, G. S.; Rocha, R. C.; Toma, H. E.; Inorg. Chim. Acta 2003, 348, 50 .
23. Handbook of X-ray data, Zschornack, G., org.; Springer-Verlag, Berlin, 2007.

24. De Souza, V. R.; Ferreira, A. M. C.; Toma, H. E.; Dalton Trans. 2003, 458.

25. Morozan, A; Jaouen, F ; Energy Environ. Sci. 2017, 5, 9269

26. Juris, A.; Balzani, V.; Barigelletti, F.; Belser, P.; von Zelewsky, A.; Coord. Chem. Rev. 1988, 84, 85 . 